Vietnam Journal of Mechanics, VAST, Vol.37, No. 1 (2015), pp. 1 -15

DOI:10.15625/0866-7136/37/1/3764

\title{
IDENTIFICATION OF MATERIAL PARAMETERS BASED ON A HOMOGENIZATION METHOD USING EBSD DATA
}

\author{
Phan Van Tung \\ Duy Tan University, Da Nang, Vietnam \\ National University of Singapore, Singapore \\ E-mail: phanvantung@dtu.edu.vn \\ Received March 18, 2014
}

\begin{abstract}
In this paper, an optimized homogenization method using uniaxial tensile tests to estimate material parameters in the micromechanical model of a heat treated DC04 steel is introduced. The method is based on a representative element model for the macroscopically homogeneous material. A Taylor polycrystal model is applied at integration points and simultaneously accounting for experimental electron backscatter diffraction (EBSD) data. Computational macro stress-strain curves are compared to experimental stress-strain curves to estimate the parameters of the DC04 steel at the different angles to rolling direction $(\mathrm{RD})$.
\end{abstract}

Keywords: Heat treated DC04 steel, elastoviscoplastic material, finite element method, Taylor type polycrystal model, EBSD texture data, MTEX toolbox.

\section{INTRODUCTION}

Most of metals used in industrial applications are polycrystalline materials. As observed in experimental micrograph, they are the set of grains with identified grain boundaries. Each grain is the aggregate of single crystals having approximately homogenous orientation. In crystallography, the crystal structure or the arrangement of atoms is considered as a cubic shape. As studied by [1] and [2], the anisotropic plasticity of polycrystalline materials is mainly caused by non-uniform distributions of crystal orientations. Therefore, the analysis of the crystallographic texture, i.e., preferred crystal orientations, plays an important role when investigating the macroscopic material behavior. The experimental EBSD technique, known as Scanning Electron Microscope (SEM) based technique, has become a major tool in measuring crystal orientations from a polycrystal structure. One application is the use of crystal orientation data at every Gauss

(C) 2015 Vietnam Academy of Science and Technology 
integration point in finite element simulations of crystal plasticity models. For this special techniques, e.g. [3-6] are developed to determine a reduced number of individual orientations based on experimental data.

Low carbon steels is currently of important interest since they are widely used in automotive systems due to their formability and high corrosion resistance. In this paper, a low carbon DC04 steel widely used in the automotive industry is investigated. The paper aims to estimate microscopic material parameters in a large strain crystal plasticity model used for a body-centered cubic (BCC) material of the DC04 steel. The estimation is performed by using uniaxial tensile experiments and the crystal orientations related to experimental EBSD data for the DC04 steel. Here the crystallographic EBSD data obtained from the heat treated process on the DC04 steel is delivered by Institute for Applied Materials-Materials and Biomechanics (IAM-WBM, Karlsruhe Institute of Technology). This texture data implying the development of a new microstructure and the formation of a new crystallographic texture will be considered. The different numbers of single crystal orientations extracted from the raw EBSD data are used for the homogenization scheme implemented at Gauss integration points. Computational results using the two-scale plasticity model are compared with experimental tensile tests in terms of stress-strain curves to estimate the underlying material parameters. The paper is organized as follows. In Section 2, the constitutive equations of the finite elastoviscoplasticity theory are described briefly. The constitutive equations are integrated over time by means of the implicit Euler scheme. The experimental data of EBSD texture and tensile curves are introduced in Section 3. In Section 4, computational tensile stress-strain curves of tensile tests are compared with experimental results for different tensile directions in the sheet plane to estimate the material parameters. The tensile test data are provided by Institute of Forming Technology and Lightweight Construction (IUL, Technical University of Dortmund). Finally, Section 5 gives conclusions.

\section{CONSTITUTIVE EQUATIONS}

\subsection{Single crystal plasticity model}

\subsubsection{Elastic law}

In this section, an elastoviscoplastic single crystal constitutive model in the large strain crystal plasticity theory $[7,8]$ is briefly summarized. The constitutive law is implemented in the commercial ABAQUS software using the subroutine UMAT, which allows user-defined material constitutive laws to be incorporated in FE simulations. The model is based upon the assumptions of small elastic strains and finite plastic strains and rotations. Plastic deformation is assumed to be the result from distinct slip mechanisms on specific crystallographic planes. The crystal elasticity properties are assumed not to be affected by the slip mechanism. The deformation gradient is decomposed multiplicatively into an elastic part $\mathbf{F}_{\mathrm{e}}$ and a plastic part $\mathbf{F}_{\mathrm{p}}$

$$
\mathbf{F}=\mathbf{F}_{\mathrm{e}} \mathbf{F}_{\mathrm{p}}
$$

The plastic deformation $\mathbf{F}_{\mathrm{p}}$ is the plastic contribution from crystallographic slips. The elastic deformation $\mathbf{F}_{\mathrm{e}}$ accounts for the lattice distortion, which is inherently elastic. 
The Kirchhoff stress tensor in the elastic law using the elastic stiffness tensor $\mathbb{C}$ on the single cubic crystal is given by

$$
\tau=\mathrm{F}_{\mathrm{e}} \tilde{\mathbb{C}}\left[\mathrm{E}_{\mathrm{e}}\right] \mathbf{F}_{\mathrm{e}}^{\mathrm{T}} \text {. }
$$

The stiffness tensor has three independent elastic constants [9]. Green's strain tensor is defined by

$$
\mathbf{E}_{\mathrm{e}}=\left(\mathbf{C}_{\mathrm{e}}-\mathbf{I}\right) / 2 \text {, }
$$

with $\mathbf{I}$ being the 2nd-order unit tensor and the right (elastic) Cauchy-Green tensor

$$
\mathbf{C}_{\mathrm{e}}=\mathrm{F}_{\mathrm{e}}^{\mathrm{T}} \mathbf{F}_{\mathrm{e}} \text {. }
$$

2.1.2. Flow rule and hardening law

A rate-dependent flow rule specifies the time evolution of the plastic part $\mathbf{F}_{\mathrm{p}}$ of $\mathbf{F}$

$$
\dot{\mathbf{F}}_{\mathrm{p}} \mathbf{F}_{\mathrm{p}}^{-1}=\sum_{\alpha} \dot{\gamma}_{\alpha} \tilde{\mathbf{M}}_{\alpha}, \quad \dot{\gamma}_{\alpha}=\dot{\gamma}_{0} \operatorname{sgn}\left(\tau_{\alpha}\right)\left|\frac{\tau_{\alpha}}{\tau^{\mathrm{C}}}\right|^{m},
$$

where the exponent $m$ quantifies the strain-rate sensitivity of the material, $\dot{\gamma}_{0}$ is a reference rate, and $\tilde{\mathbf{M}}_{\alpha}$ is the Schmid tensor. $\tau^{C}$ denotes the critical resolved shear stress. The following rate-dependent Kocks-Mecking hardening model $[4,10]$

$$
\dot{\tau}^{C}\left(\tau_{\alpha}, \tau^{C}\right)=\Theta_{0}\left(1-\frac{\tau^{C}}{\tau_{V}^{C}\left(\tau_{\alpha}, \tau^{C}\right)}\right) \dot{\gamma}\left(\tau_{\alpha}, \tau^{C}\right),
$$

is used, where the critical Voce stress is specified by

$$
\tau_{V}^{C}\left(\tau_{\alpha}, \tau^{C}\right)=\tau_{V 0}^{C}\left(\frac{\dot{\gamma}\left(\tau_{\alpha}, \tau^{C}\right)}{\dot{\gamma}_{0}}\right)^{\frac{1}{n}}
$$

with the asymptotic critical resolved shear stress $\tau_{V 0}^{C}$ and the initial hardening modulus $\Theta_{0}$. The rate of the accumulated plastic slip is computed by

$$
\dot{\gamma}=\sum_{\alpha}\left|\dot{\gamma}_{\alpha}\left(\tau_{\alpha}, \tau^{C}\right)\right|
$$

The resolved shear stress is defined by

$$
\tau_{\alpha}=\mathbf{T}_{e}^{\prime} \cdot \tilde{\mathbf{M}}_{\alpha}
$$

where

$$
\mathrm{T}_{\mathrm{e}}=\mathrm{C}_{\mathrm{e}} \mathrm{S}_{\mathrm{e}}^{2 \mathrm{PK}} \text {, }
$$

denotes the Mandel stress tensor. The second Piola-Kirchhoff in the undistorted state is given by $\mathbf{S}_{\mathrm{e}}^{2 \mathrm{PK}}=J \mathrm{~F}_{\mathrm{e}}^{-1} \tau \mathrm{F}_{\mathrm{e}}^{-\mathrm{T}} . J=\operatorname{det}\left(\mathrm{F}_{\mathrm{e}}\right)$ is the determinant of $\mathbf{F}_{\mathrm{e}}$. The Schmid tensors are defined in terms of the slip direction $\tilde{\mathbf{d}}_{\alpha}$ and slip plane normal $\tilde{\mathbf{n}}_{\alpha}$ in the undistorted configuration

$$
\tilde{\mathbf{M}}_{\alpha}=\tilde{\mathbf{d}}_{\alpha} \otimes \tilde{\mathbf{n}}_{\alpha} .
$$

The initial conditions for the ordinary differential equation are $\mathbf{F}_{\mathrm{e}}(0)=\mathbf{Q}(t=0) \in S O(3)$ and the initial critical resolved shear stress $\tau^{C}(0)=\tau_{0}^{C}$. The crystal orientation is given by a proper orthogonal tensor $\mathbf{Q}(t)=\mathbf{g}_{i}(t) \otimes \mathbf{e}_{i}$, where the vectors $\mathbf{g}_{i}$ and $\mathbf{e}_{i}$ denote the 
orthonormal lattice vectors and the fixed orthonormal basis, respectively. The initial orientation of the single crystal $\mathbf{Q}(t=0)=\mathbf{g}_{i}(0) \otimes \mathbf{e}_{i}$ is defined in terms of the orthonormal lattice vectors $\mathbf{g}_{i}(0)$ at the time $t=0$. For body-centered cubic (BCC) slip sytems of DC04 steel, the attention is focused on a combination of $\{110\}\langle 111\rangle$ and $\{112\}\langle 111\rangle$ slip system families [11]. There are two slip directions in each of the slip planes along the main diagonals of the cube. In total, there are 24 slip systems [11,12].

\subsection{Taylor type polycrystal model}

The crystallographic texture of polycrystalline materials is known to be of significant importance for the sheet metal forming behavior. Taylor type polycrystal models known as numerically the most effective two-scale models take into account the crystallographic texture at integration Gauss points. In general, Taylor models, e.g., [13-17] assume that the deformation gradient field is homogeneous for all grains through the microstructure of the polycrystal. The macroscopic deformation gradient is equal to the volume average of the microscopic deformation gradient

$$
\overline{\mathbf{F}}=\frac{1}{V} \int_{V} \mathbf{F} \mathrm{d} V,
$$

with respect to the reference configuration. The effective Kirchhoff stress tensor is computed as the volume average of crystal stress with respect to the current configuration

$$
\bar{\tau}=\frac{1}{V} \int_{V} \tau \mathrm{d} V=\sum_{\beta}^{M} v_{\beta} \tau_{\beta},
$$

where $M$ is the total number of grains in the discretized crystallographic texture, $v_{\beta}$ is the volume fraction of the grain $\beta$ and $\tau_{\beta}$ is the corresponding Kirchhoff stress tensor.

\section{EXPERIMENTAL DATA}

\subsection{EBSD crystallographic texture data}

\subsubsection{EBSD technique}

In recent years, EBSD technique [18] has become an important technique for the quantitative characterization of different microstructural properties such as the grain size, the grain boundary structure, and the orientation distribution. This technique allows to obtain spatially resolved crystallographic information by a Scanning Electron Microscope (SEM). For every point analyzed on a sample, the position, the phase and the crystallographic orientation are stored. A specimen of DC04 steel is investigated in the cold formed and heat treated state by using the EBSD technique to obtain a corresponding database and simultaneously to identify a two-dimensional approximation of the grain structure. The software package MTEX [19,20], a Matlab toolbox developed since 1997, is used for the quantitative analysis of experimental textures. The obtained EBSD database is processed by MTEX to identify the grains and their boundaries. In order to identify a grain from the EBSD measurement, it is necessary to determine its grain boundary and the average orientation inside the grain. These identifications can be handled by the open-source texture toolbox MTEX in terms of the misorientation over the set of all measurement points inside a grain. The heat treated EBSD microstructure and 
the clustered heat treated EBSD microstructure are shown in Fig. 1. After the clustering process in MTEX, grain boundaries are identified and the total number of the identified grains are found to be 2554. An output database is shown in Tab. 1, including three Euler angles of clustered grains and their corresponding pixels.

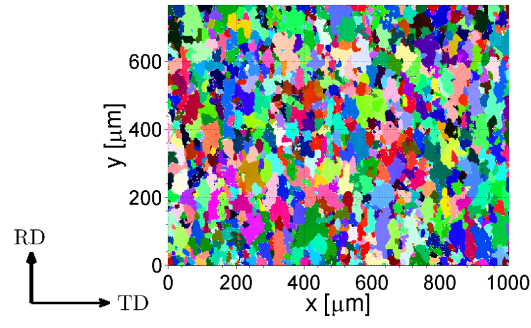

(a) Raw EBSD data set

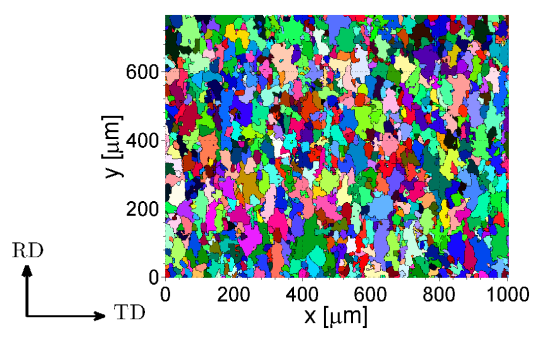

(b) Clustered EBSD data set

Fig. 1. Microstructural EBSD images of the heat treated DC04 steel

Table 1. The data set for identification of grains in the clustered heat treated specimen of DC04 steel

\begin{tabular}{|c|c|c|c|c|}
\hline Grain & Euler1 & Euler2 & Euler3 & Pixels \\
\hline 1 & 83.32 & 47.374 & 23.528 & 3 \\
2 & 117.36 & 47.152 & 37.653 & 4 \\
3 & -92.53 & 38.297 & 82.924 & 1 \\
4 & 25.048 & 127.55 & 158.2 & 1 \\
5 & -15.257 & 46.542 & 37.09 & 6 \\
$\vdots$ & $\vdots$ & $\vdots$ & $\vdots$ & $\vdots$ \\
2550 & 0.74703 & 14.359 & 11.114 & 71 \\
2551 & -113.42 & 43.691 & 47.862 & 443 \\
2552 & -151.99 & 44.525 & 71.867 & 7 \\
2553 & -157.13 & 42.302 & 62.368 & 21 \\
2554 & -30.854 & 41.545 & 34.8 & 72 \\
\hline
\end{tabular}

\subsubsection{Low dimensional description of crystallographic texture}

In this section, an effective method is introduced for the selection of representative grain orientations based on discrete texture data. The reduced orientation distribution has to reproduce the overall crystallographic texture of the investigated material in a statistical as well as mechanical sense. The crystal orientation distribution function (CODF) represents the crystallographic texture in terms of a volume fraction description of crystal 
orientations. Mathematically, it represents the volume fraction of crystals with orientation $\mathbf{Q} \in S O(3)$, i.e.,

$$
\frac{\mathrm{d} V}{V}=f(\mathbf{Q}) \mathrm{d} Q \text {. }
$$

For a random texture $f(\mathbf{Q}) \equiv 1$ holds. The orientation of a single crystal in a polycrystalline structure can be uniquely determined by the specification of the rotation $Q \in$ $S O$ (3) which maps the sample fixed basis vectors $\mathbf{e}_{i}$ onto the lattice vectors $\mathbf{g}_{i}$ by $\mathbf{g}_{i}=\mathbf{Q} \mathbf{e}_{i}$. The rotation $\mathbf{Q}$ is commonly parameterized by a triple of Euler angles $\phi_{1}, \Phi, \phi_{2}$ in the 'zxz'-convention and is represented by a $3 \times 3$ orthogonal matrix [2] given by

$$
\begin{aligned}
Q_{i j} & =\left[\begin{array}{ccc}
\cos \phi_{1} & -\sin \phi_{1} & 0 \\
\sin \phi_{1} & \cos \phi_{1} & 0 \\
0 & 0 & 1
\end{array}\right]\left[\begin{array}{ccc}
1 & 0 & 0 \\
0 & \cos \Phi & -\sin \Phi \\
0 & \sin \Phi & \cos \Phi
\end{array}\right]\left[\begin{array}{ccc}
\cos \phi_{2} & -\sin \phi_{2} & 0 \\
\sin \phi_{2} & \cos \phi_{2} & 0 \\
0 & 0 & 1
\end{array}\right] \\
& =\left[\begin{array}{ccc}
\cos \phi_{1} \cos \phi_{2}-\sin \phi_{1} \cos \Phi \sin \phi_{2} & -\cos \phi_{1} \sin \phi_{2}-\sin \phi_{1} \cos \Phi \cos \phi_{2} & \sin \Phi \sin \phi_{1} \\
\sin \phi_{1} \cos \phi_{2}+\cos \phi_{1} \cos \Phi \sin \phi_{2} & -\sin \phi_{1} \sin \phi_{2}+\cos \phi_{1} \cos \Phi \cos \phi_{2} & -\sin \Phi \cos \phi_{1} \\
\sin \Phi \sin \phi_{2} & \sin \Phi \cos \phi_{2} & \cos \Phi
\end{array}\right],
\end{aligned}
$$

where the infinitesimal volume element $\mathrm{d} Q=\frac{1}{8 \pi^{2}} \sin (\Phi) \mathrm{d} \phi_{1} \mathrm{~d} \phi_{2} \mathrm{~d} \Phi$. In order to save the time-consuming of numerical FE computations, the number of grains needs to be reduced but simultaneously has to represent the crystallographic texture data accurately. Reduced crystallographic textures including only 200 grains, 400 grains and 650 grains with the corresponding largest volume fractions are extracted and used for the numerical homogenization. Considering the case of a cubic crystal symmetry, an orthotropic orientation data set needs to be used. The orthotropic data can be obtained by rotating counterclockwise the grain orientation data set 180 degrees in turn about the orthonormal axes in the lattice system. The orthogonal rotations characterized by three orthogonal matrices with respect to the orthonormal crystal lattice vectors $\left\{\mathbf{g}_{i}\right\}(i=1 \ldots 3)$ are given by

$$
\begin{aligned}
& \mathbf{R}_{1}\left(180^{\circ}\right)=\left[\begin{array}{ccc}
1 & 0 & 0 \\
0 & -1 & 0 \\
0 & 0 & -1
\end{array}\right], \\
& \mathbf{R}_{2}\left(180^{\circ}\right)=\left[\begin{array}{ccc}
-1 & 0 & 0 \\
0 & 1 & 0 \\
0 & 0 & -1
\end{array}\right], \\
& \mathbf{R}_{3}\left(180^{\circ}\right)=\left[\begin{array}{ccc}
-1 & 0 & 0 \\
0 & -1 & 0 \\
0 & 0 & 1
\end{array}\right] .
\end{aligned}
$$

Each multiplication of an orthogonal rotation matrix $\mathbf{R}_{i}(i=1 \ldots 3)$ and each grain orientation $\mathbf{Q}$, given by $\mathbf{R}_{i} \mathbf{Q}(i=1 \ldots 3)$, generates an additional grain orientation. This means that the orthotropic orientation data set is four times larger than the initial set. Therefore, the orthotropic orientation data sets of 2554 grains (the raw data), 200 grains, 400 grains and 605 grains will consist of 10216 orientations, 800 orientations, 1600 orientations and 
2420 orientations, respectively. Here, two important characteristics for the graphical representation of the texture data are to be evaluated. The first is the pole figure which is used to plot a set of poles for corresponding grain orientations based on stereographic projections in 3D space. The second is the aforementioned CODF. The $\{111\},\{100\}$ and $\{110\}$ pole figures and the CODF of the orthotropic orientation data sets (10216 orientations and 800 orientations) are shown in Figs. 2 and 3. In Fig. 3, the CODFs are determined based on the kernel distribution of von Mises Fisher in the MTEX algorithm . It can be seen that the pole figures and the CODF of 10216 and 800 grains, respectively, are approximately similar.
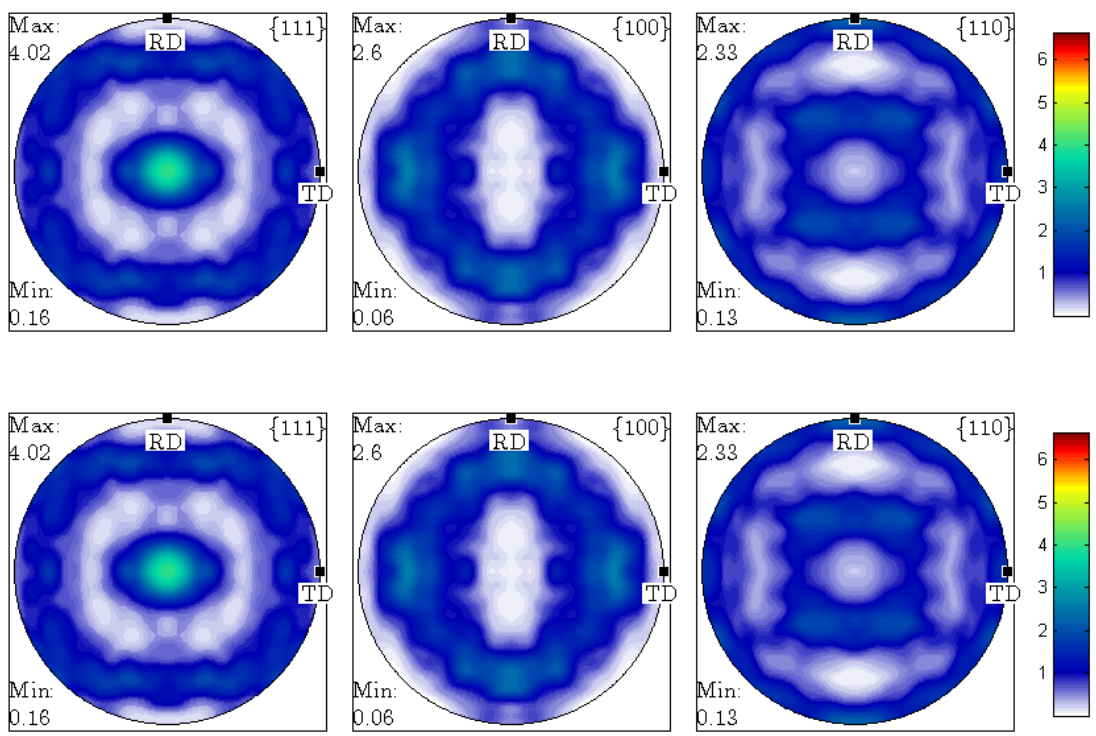

Fig. 2. Comparison between pole figures of 10216 grains (above) and 800 grains (below)
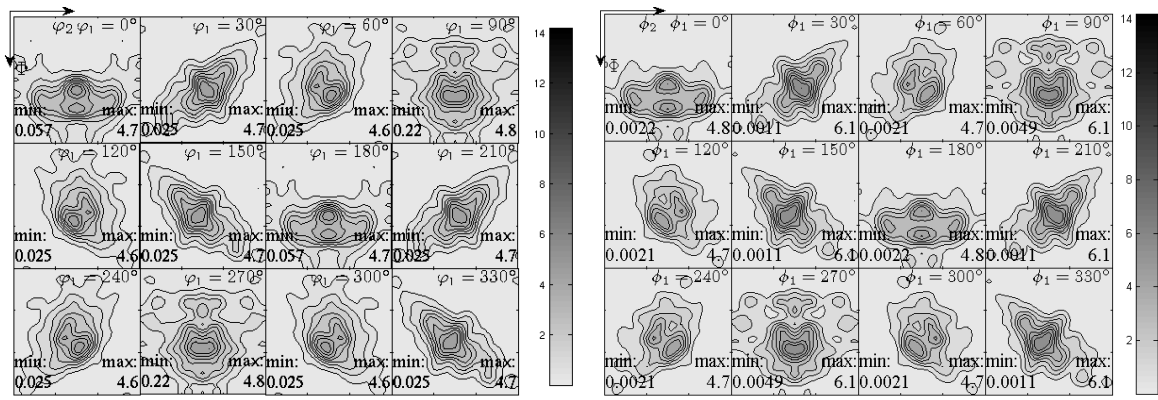

Fig. 3. Slices of CODF: 10216 grains (left) and 800 grains (right) 

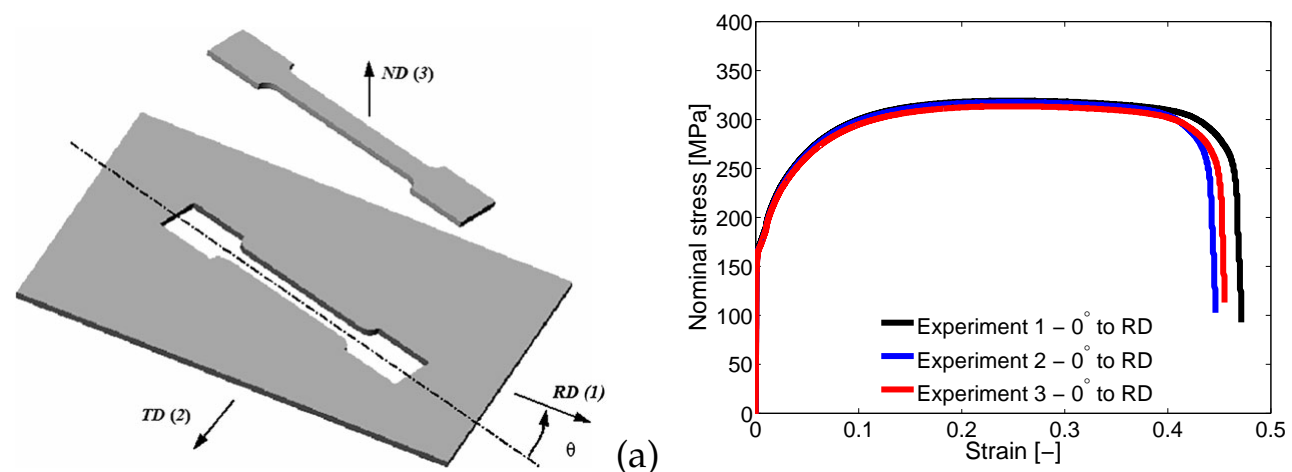

(b)
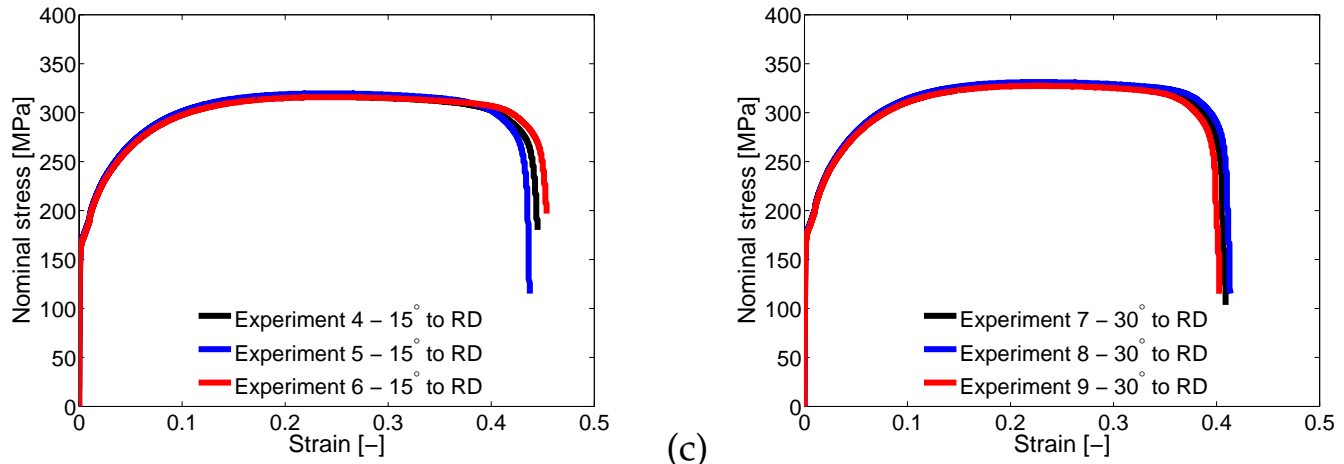

(d)
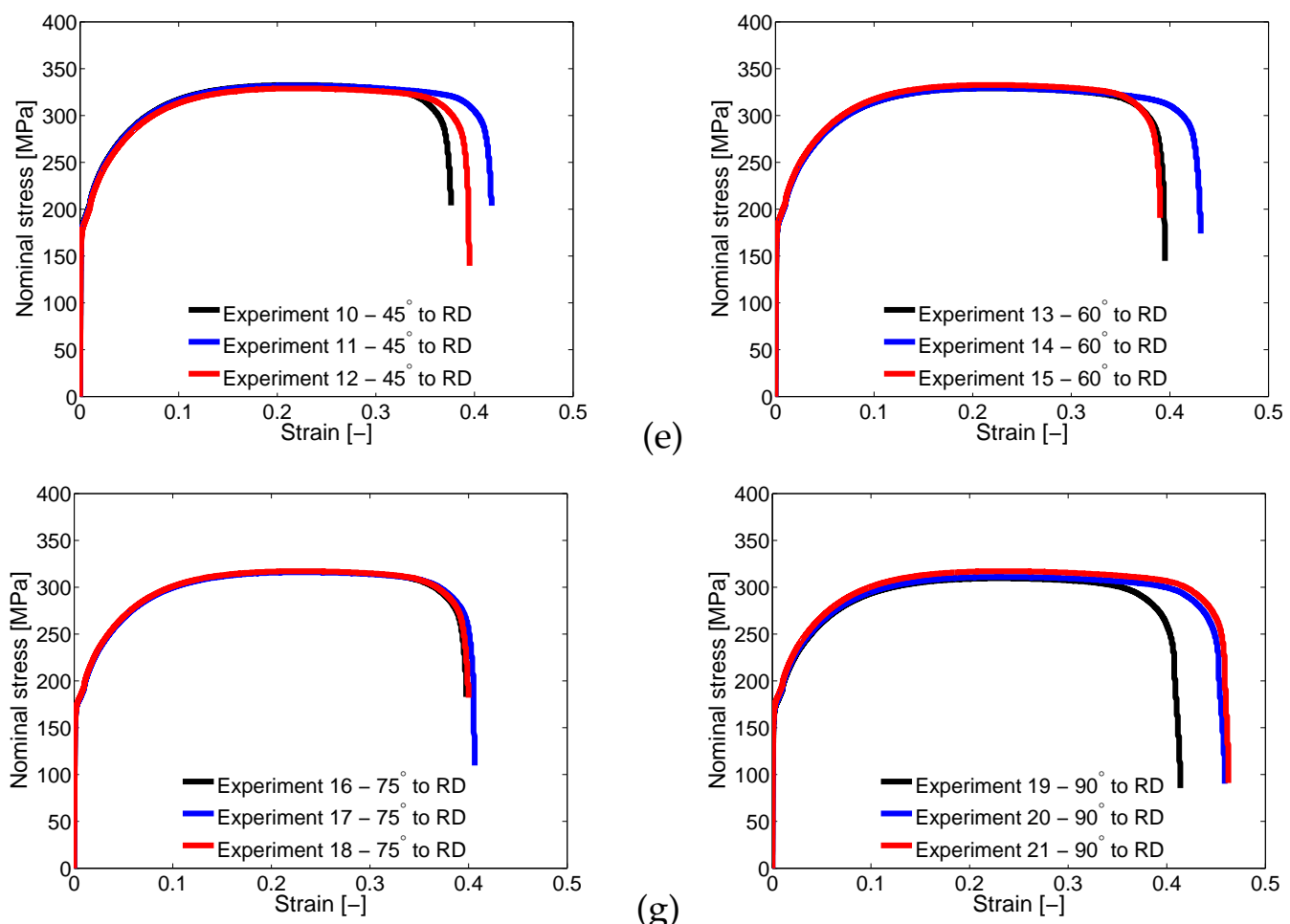

(e)

$(\mathrm{g})$

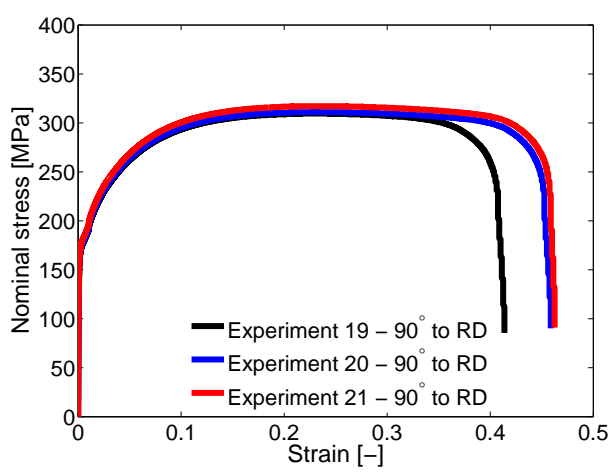

(h)

Fig. 4. (a) Tensile specimen at different angles to RD [21] and experimental stress-strain curves for different angles to RD - (b) $0^{\circ}$, (c) $15^{\circ}$, (d) $30^{\circ}$, (e) $45^{\circ}$, (f) $60^{\circ}$, (g) $75^{\circ}$, (h) $90^{\circ}$. 


\subsection{Tensile curves}

The most widely used basic test of sheet metal forming is the uniaxial tensile test. As illustrated in Fig. 4 (a), a specimen cut from a heat treated sheet metal of a DC04 steel at different angles to the rolling direction (RD) is used to carry out uniaxial tensile tests. The uniaxial tensile experiment performed by Institute of Forming Technology and Lightweight Construction (IUL, Technical University of Dortmund) is used to investigate the macroscopic stress-strain relation. Several data sets are identified for specimens oriented at $0^{\circ}, 15^{\circ}, 30^{\circ}, 45^{\circ}, 60^{\circ}, 75^{\circ}$ and $90^{\circ}$ to the RD. The initial thickness of the specimen varies in the range $t_{0}=1.16-1.2 \mathrm{~mm}$ with a gauge length $L_{0}=80 \mathrm{~mm}$ and the range $w_{0}=19.81-19.92 \mathrm{~mm}$. The tests were conducted at the constant velocity of $0.0025 \mathrm{~s}^{-1}$. These experiments contain information of the displacements and the forces over time. The nominal stress is calculated by $\sigma_{0}=F / A_{0}$, where $F$ is the force and $A_{0}=t_{0} w_{0}$ is the initial area of the cross section. The engineering strain is given by $\varepsilon=L(t) / L(0)-1=\Delta L(t) / L(0)$. Figs. $4(\mathrm{~b}-\mathrm{h})$ shows the nominal stress versus the engineering strain for differently oriented specimens. It can be seen that there are three tensile experiments performed at each fixed angle $\theta$ to RD. To make it simple, a mean stress-strain curve is computed based on these three experimental curves in the range of strain $0-0.2$.

\section{ESTIMATION OF MATERIAL MICRO-PARAMETERS BASED ON TENSILE SIMULATIONS}

This section aims to identify the material parameters used in the micromechanical model of DC04 steel based on uniaxial tensile tests. A FE model for modeling the tensile tests is constructed by ABAQUS/CAE and the Taylor type polycrystal model is applied at the integration points of finite elements. The mechanical constitutive law discussed in Section 2 is implemented numerically by a user material behavior (UMAT subroutine). The orthotropic orientation data sets (800 grains, 1600 grains and 2420 grains) of the heat treated DC04 steel will be the input data used in the Taylor type polycrystal model as initial grain orientation distribution. In Fig. 5, the FE model representing a representative element (8-node linear brick element type - C3D8) for the macroscopically homogeneous material is shown. Stresses and strains are computed at 8 integration Gauss points within the FE model. The length, the width and the area are $1 \mathrm{~mm}, 1 \mathrm{~mm}$ and $1 \mathrm{~mm}^{2}$, respectively. The initial time increment is $10^{-6} \mathrm{~s}$ and the maximum time increment is $1 \mathrm{~s}$. The total time in the simulations is $200 \mathrm{~s}$ corresponding to the final displacement of $u_{x}=0.2$. As a result, the applied strain rate is $10^{-3} \mathrm{~s}^{-1}$. Note that each rotation of the initial orientation data set about the normal direction $\mathbf{e}_{3}$ (ND) with angle $\theta$ corresponds to the oriented specimen. The rotation matrix $\underline{\underline{\Theta}}$ representing the rotation about $\mathbf{e}_{3}$, can be expressed as follows

$$
\underline{\underline{\Theta}}(\theta)=\left[\begin{array}{ccc}
\cos (\theta) & -\sin (\theta) & 0 \\
\sin (\theta) & \cos (\theta) & 0 \\
0 & 0 & 1
\end{array}\right] .
$$

Each tensile simulation for a fixed angle $\theta$ is performed for the orthotropic orientation set. The nominal stress is computed by $\sigma_{0}=F / A_{0}$, where $F$ is the sum of computed 


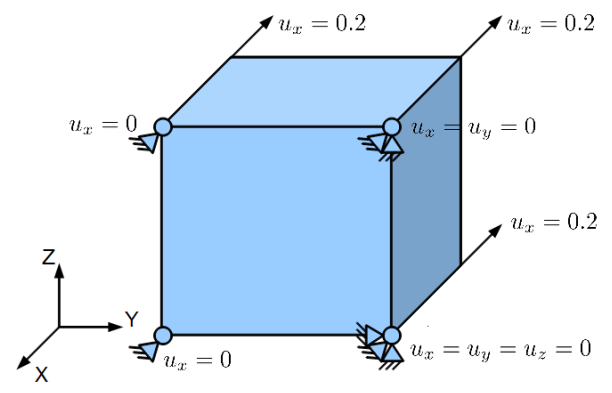

Fig. 5. FE-model of the uniaxial tensile test with one finite element.

forces at nodes along the tensile direction during deformation. In the following investigation, a set of material parameters are estimated by comparing simulation results to experimental data. This estimation will be done based on stress-strain curves and the least square fitting method. The elastic constants of DC04 steel used in tensile test simulations are explained in Section 2.1. The other material parameters, i.e., the reference slip rate $\dot{\gamma}_{0}$, the strain-rate sensitivity parameter $m$, the initial critical resolved shear stress $\tau_{0}^{C}$, the asymptotic critical resolved shear stress $\tau_{V 0}^{C}$, and the initial hardening modulus $\Theta_{0}$ are estimated based on the numerical stress-strain curves. Firstly, the values of the initial and asymptotic critical resolved shear stresses are estimated via the Taylor factor $M \cong 3$ between the microscopic shear stress and the macroscopic nominal stress

$$
\left.\tau_{0}^{C} \cong \frac{\sigma_{0}^{\text {macro }}}{M}\right|_{\theta=0^{\circ}},\left.\quad \tau_{V 0}^{C} \cong \frac{\sigma_{\infty}^{\text {macro }}}{M}\right|_{\theta=0^{\circ}},
$$

where the macroscopic nominal stresses $\sigma_{0}^{\text {macro }}$ and $\sigma_{\infty}^{\text {macro }}$ can be estimated from the experimental tensile curves. Secondly, these two microscopic shear stresses and the hardening modulus $\Theta_{0}$ are adjusted to fit to the experimental curves for all different angles $\theta$. The least square method is applied in order to determine the optimal parameters. The error is defined by

$$
E_{2}=\sqrt{\frac{1}{n} \sum_{i=1}^{n} d_{i}^{2}}=\sqrt{\frac{1}{n} \sum_{i=1}^{n}\left|P_{i}^{\text {exp }}-P_{i}^{\text {num }}\right|},
$$

where $n$ is the number of data points and $d_{i}$ is the distance between the experimental data point $P_{i}^{e x p}$ and simulated data point $P_{i}^{\text {num }}$ with respect to the same strain. By using this optimal set, the comparison between the experimental and numerical results using the different orthotropic data sets for the oriented specimen at different angles to RD is depicted in Figs. 6, 7 and 8. It can be seen that these numerical and experimental tensile curves showed a good match when applying different numbers of grain orientations at the integration points. The 200-orientation data set extracted from the raw data set is sufficient to represent the crystallographic texture data accurately. Tab. 2 shows the set of optimal material parameters identified based on the minimization of $E_{2}$ for all tensile directions simultaneously. 


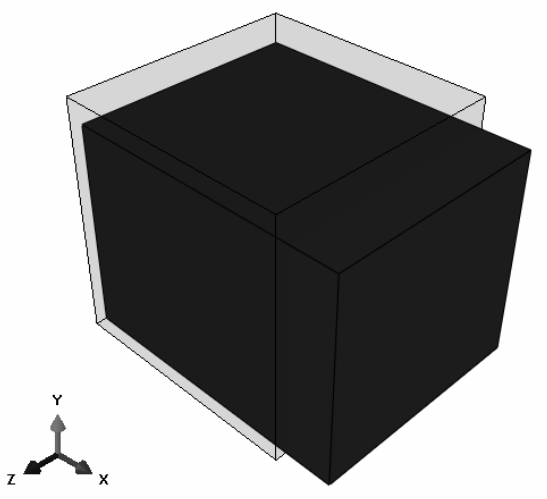

(a)

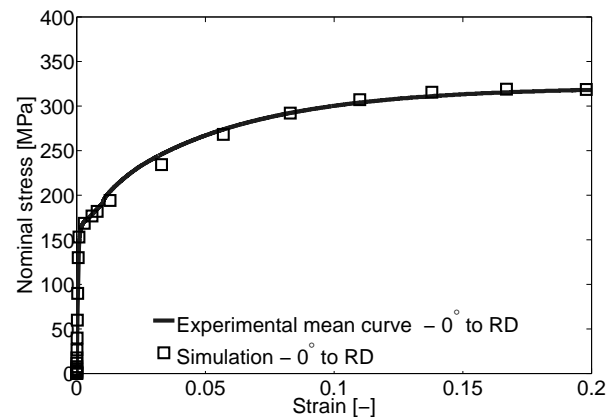

(b)
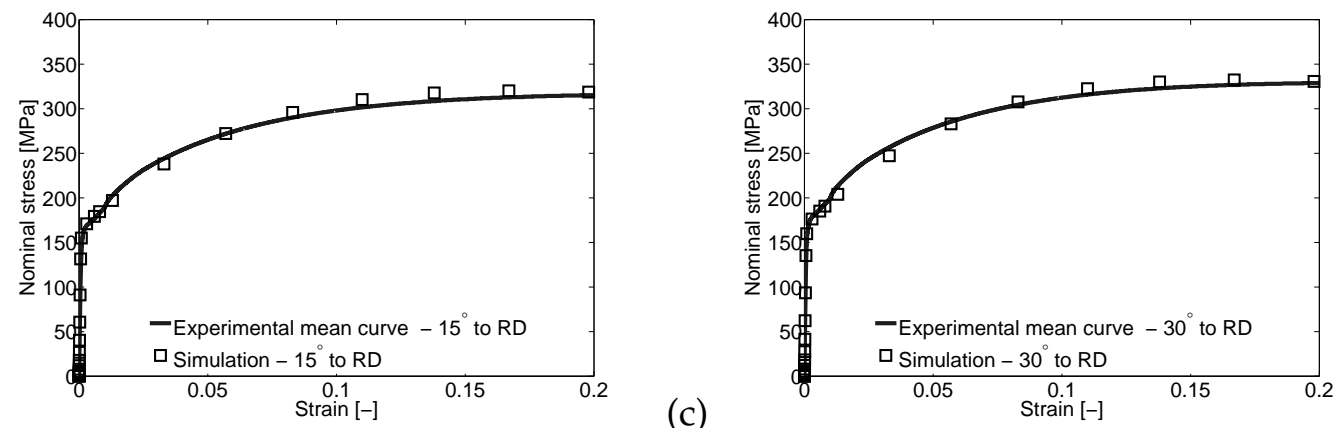

(d)
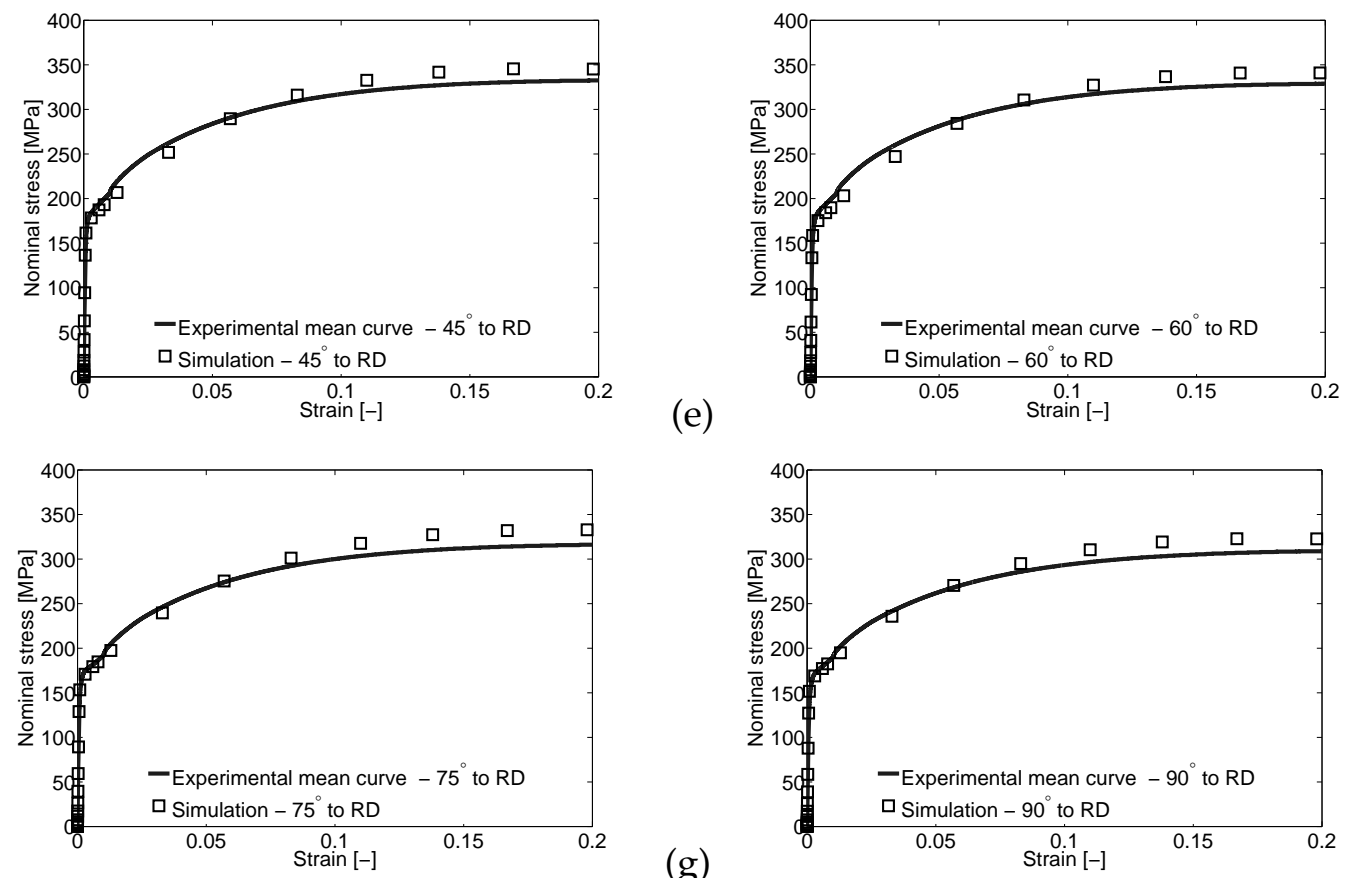

(e)

(g)

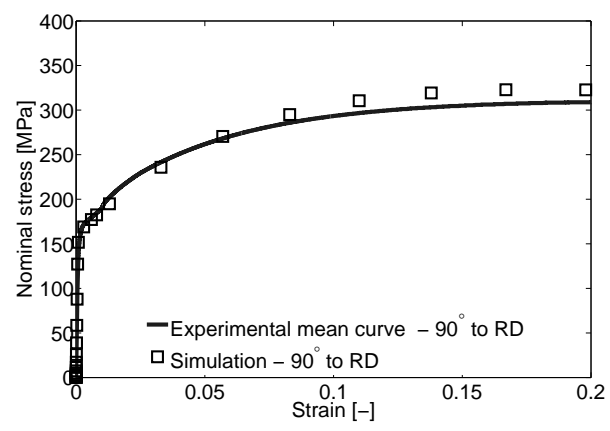

(h)

Fig. 6. (a) Undeformed (shade color) and deformed (black color) configuration of the representative element at the end state of FE simulation and numerically determined stress-strain curves using 800 grains in comparison to experimental data for different angles to RD - (b) $0^{\circ}$, (c) $15^{\circ}$, (d) $30^{\circ}$, (e) $45^{\circ}$, (f) $60^{\circ}$, (g) $75^{\circ}$, (h) $90^{\circ}$ 


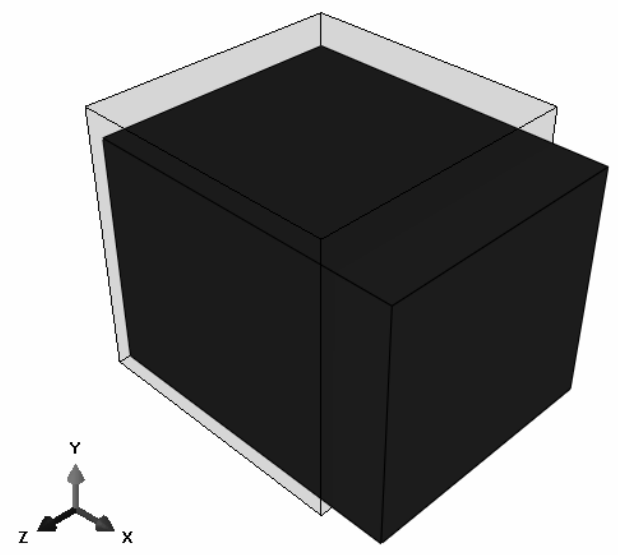

(a)
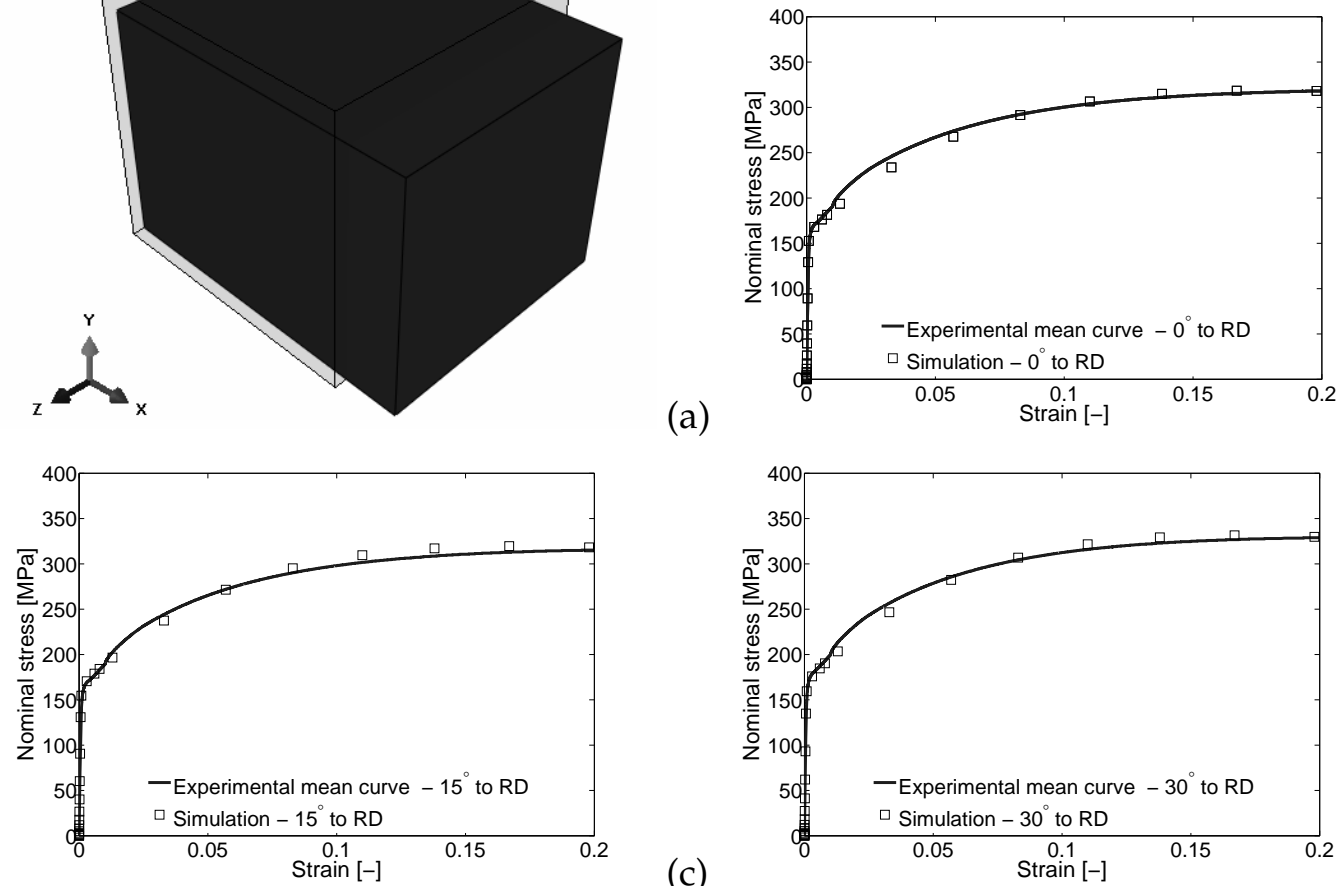

(b)

(c)

(d)
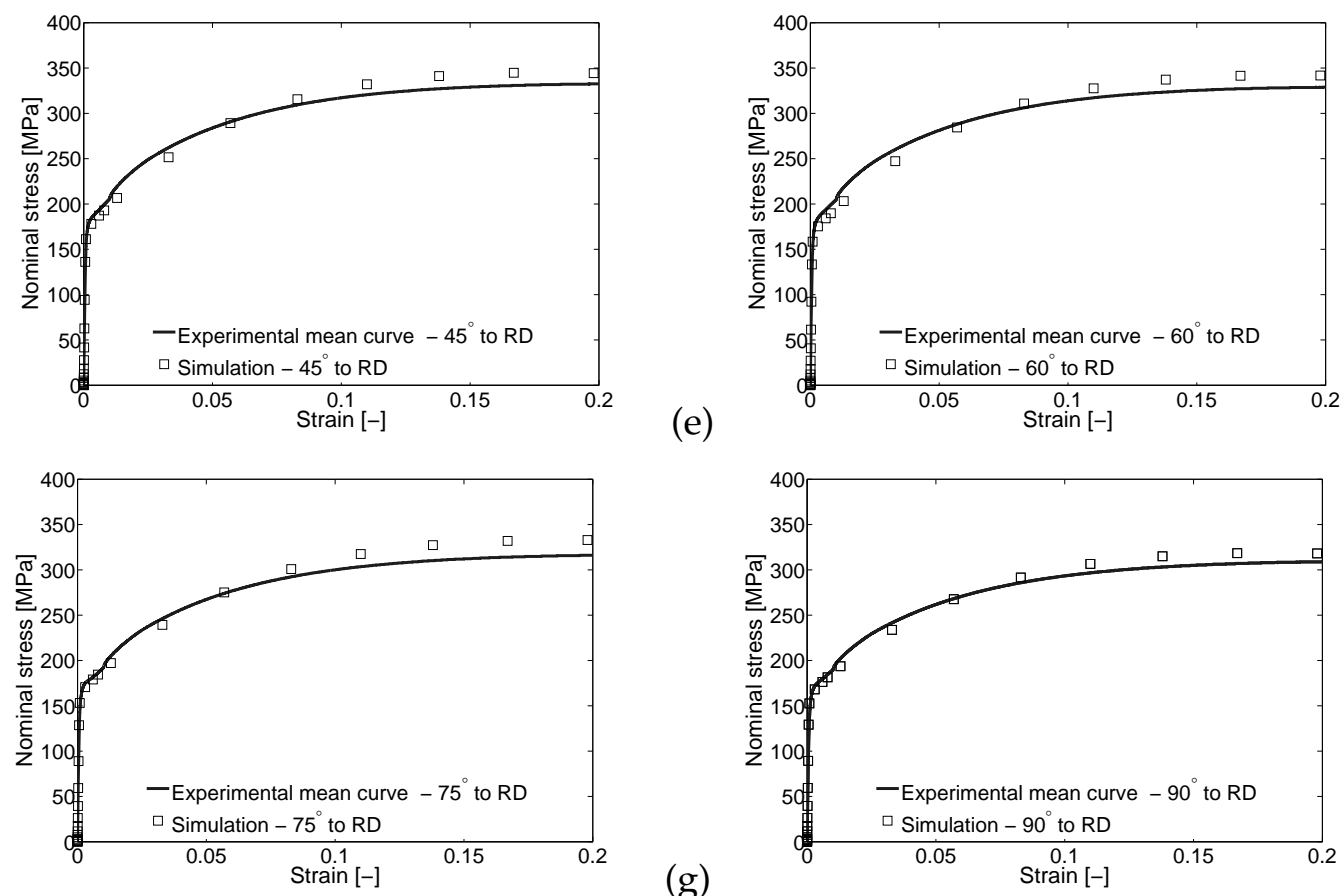

(h)

Fig. 7. (a) Undeformed (shade color) and deformed (black color) configuration of the representative element at the end state of FE simulation and numerically determined stress-strain curves using 1600 grains in comparison to experimental data for different angles to RD - (b) $0^{\circ}$, (c) $15^{\circ}$, (d) $30^{\circ}$, (e) $45^{\circ}$, (f) $60^{\circ}$, (g) $75^{\circ}$, (h) $90^{\circ}$ 


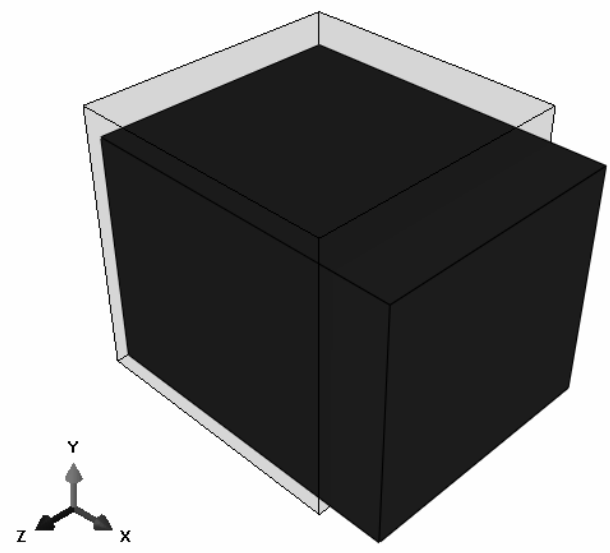

(a)
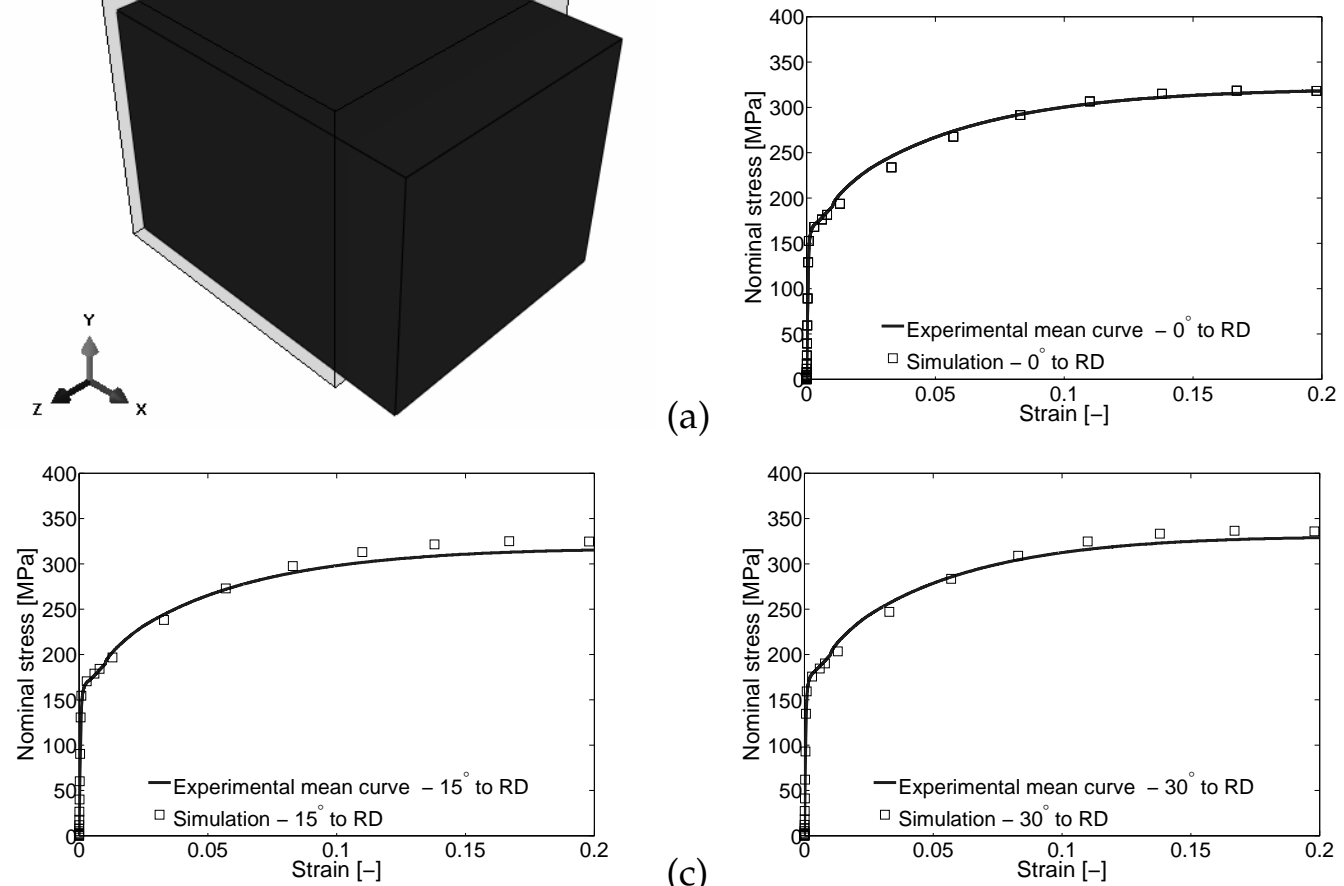

(b)

(c)

(d)
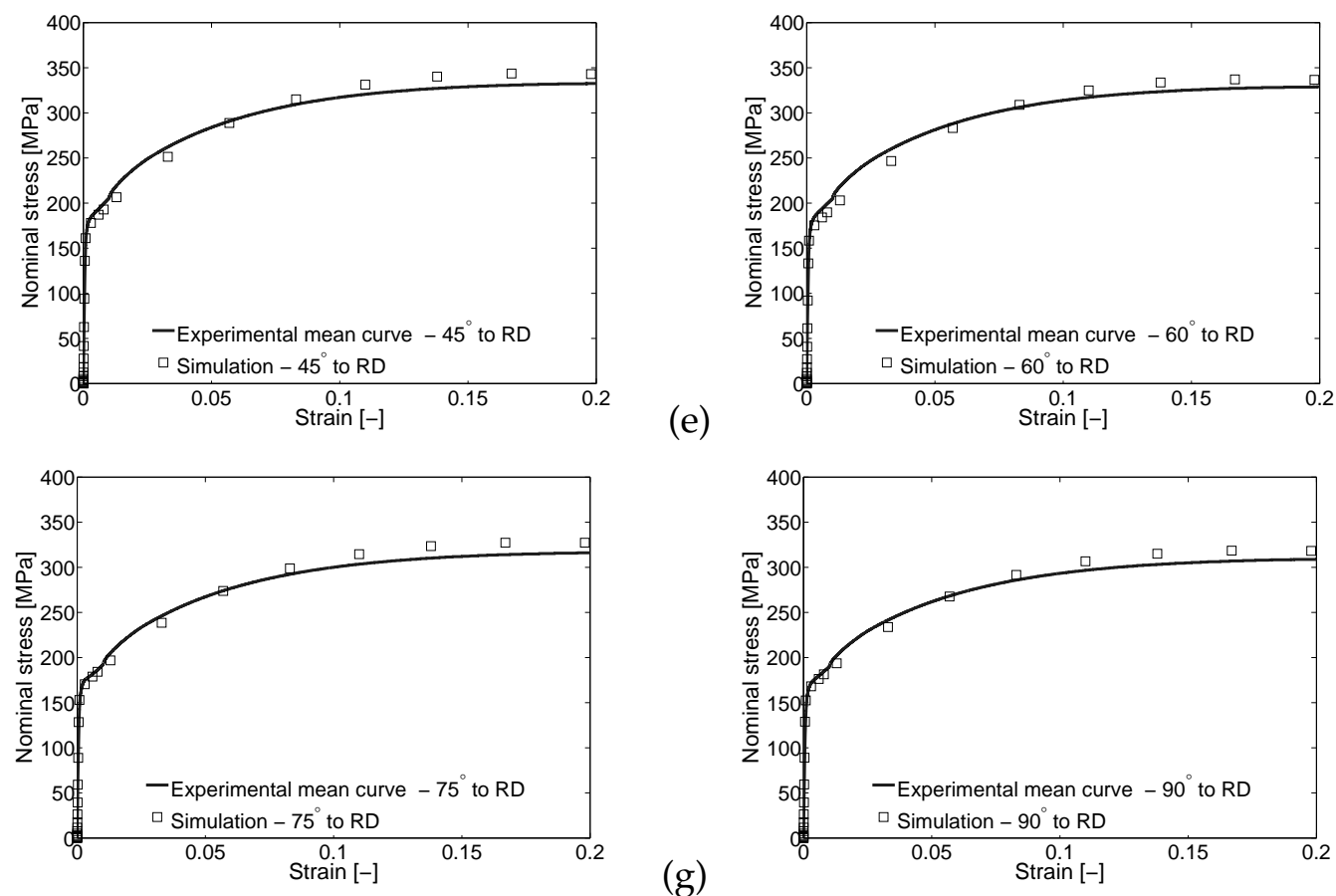

(h)

Fig. 8. (a) Undeformed (shade color) and deformed (black color) configuration of the representative element at the end state of FE simulation and numerically determined stress-strain curves using 2420 grains in comparison to experimental data for different angles to RD - (b) $0^{\circ}$, (c) $15^{\circ}$, (d) $30^{\circ}$, (e) $45^{\circ}$, (f) $60^{\circ}$, (g) $75^{\circ}$, (h) $90^{\circ}$ 
Table 2. Set of identified material parameters based on the experimental tensile tests

\begin{tabular}{|c|c|c|}
\hline$C_{1111}[\mathrm{GPa}]$ & $C_{1122}[\mathrm{GPa}]$ & $C_{1212}[\mathrm{GPa}]$ \\
\hline 231.5 & 135.0 & 116.0 \\
\hline
\end{tabular}

Elastic constants of DC04 steel [22]

\begin{tabular}{|c|c|c|c|c|c|}
\hline$m[-]$ & $\dot{\gamma}_{0}\left[\mathrm{~s}^{-1}\right]$ & $n[-]$ & $\tau_{0}^{C}[\mathrm{MPa}]$ & $\tau_{V 0}^{C}[\mathrm{MPa}]$ & $\Theta_{0}[\mathrm{MPa}]$ \\
\hline 20 & 0.001 & 5 & 67 & 130 & 755 \\
\hline
\end{tabular}

Material parameters in flow rule and hardening law

\section{CONCLUSION}

In this paper, a finite strain crystal plasticity model for BCC materials of DC04 steel has been implemented in ABAQUS. A comparison of the crystal plasticity FE simulation with the experimental tensile test for the stress-strain curves has been presented at different angles to RD. The macroscopic material behaviour in tensile tests has been used to determine an optimal set of material parameters in the micromechanical model using the reduced texture data of the heat treated DC04 steel. The different numbers of single crystal orientations have been extracted from experimental EBSD data. The mechanical constitutive equations of a large strain crystal plasticity model for the steel and BCC slip mechanisms in the coupling of $\{110\}+\{112\}\langle 111\rangle$ slip systems have been applied for these simulations. The elastic constants for the DC04 steel were taken from literature. The study illustrated how the crystallographic information could be incorporated into a continuum mechanical modeling of a basic sheet metal forming test.

\section{ACKNOWLEDGEMENTS}

The provision of heat treated DC04 steel EBSD data by Institute for Applied MaterialsMaterials and Biomechanics (IAM-WBM, KIT) is acknowledged. The author also would like to thank the Institute of Forming Technology and Lightweight Construction (TU Dortmund) for providing the tensile experiments. This work was partially supported by Vietnam National Foundation for Science and Technology Development (NAFOSTED) through a grant number 103.99-2012.13 to the Institute of Research and Development at Duy Tan University (Vietnam).

\section{REFERENCES}

[1] E. H. Lee. Elastic-plastic deformation at finite strains. Journal of Applied Mechanics, 36, (1), (1969), pp. 1-6.

[2] H. J. Bunge. Texture analysis in materials science. Culliver Verlag Göttingen, (1993).

[3] D. Raabe, P. Klose, B. Engl, K. P. Imlau, F. Friedel, and F. Roters. Concepts for integrating plastic anisotropy into metal forming simulations. Advanced Engineering Materials, 4, (4), (2002), pp. 169-180. 
[4] T. Böhlke, G. Risy, and A. Bertram. A texture component model for anisotropic polycrystal plasticity. Computational Materials Science, 32, (3), (2005), pp. 284-293.

[5] G. Rousselier, M. Luo, and D. Mohr. Macroscopic plasticity modeling of anisotropic aluminum extrusions using a Reduced Texture Methodology. International Journal of Plasticity, 30, (2012), pp. 144-165.

[6] K. Joechen and T. Böhlke. Representative reduction of crystallographic orientation data. Journal of Applied Crystallography, 46, (4), (2013), pp. 960-971.

[7] R. J. Asaro and A. Needleman. Overview no. 42 Texture development and strain hardening in rate dependent polycrystals. Acta Metallurgica, 33, (6), (1985), pp. 923-953.

[8] C. A. Bronkhorst, S. R. Kalidindi, and L. Anand. Polycrystalline plasticity and the evolution of crystallographic texture in FCC metals. Philosophical Transactions of the Royal Society of London. Series A: Physical and Engineering Sciences, 341, (1662), (1992), pp. 443-477.

[9] T. C. T. Ting and C. O. Horgan. Anisotropic elasticity: Theory and applications, Vol. 405. Oxford University Press, New York, (1996).

[10] U. F. Kocks and H. Mecking. Physics and phenomenology of strain hardening: the FCC case. Progress in Materials Science, 48, (3), (2003), pp. 171-273.

[11] T. Yalcinkaya, W. A. M. Brekelmans, and M. G. D. Geers. BCC single crystal plasticity modeling and its experimental identification. Modelling and Simulation in Materials Science and Engineering, 16, (8), (2008), pp. doi:10.1088/0965-0393/16/8/085007.

[12] A. Paquin, S. Berbenni, V. Favier, X. Lemoine, and M. Berveiller. Micromechanical modeling of the elastic-viscoplastic behavior of polycrystalline steels. International Journal of Plasticity, 17, (9), (2001), pp. 1267-1302.

[13] G. I. Taylor. Plastic strain in metals. J. Inst. Metals, 62, (1938), pp. 307-320.

[14] K. K. Mathur and P. R. Dawson. On modeling the development of crystallographic texture in bulk forming processes. International Journal of Plasticity, 5, (1), (1989), pp. 67-94.

[15] T. Böhlke, G. Risy, and A. Bertram. Finite element simulation of metal forming operations with texture based material models. Modelling and Simulation in Materials Science and Engineering, 14, (3), (2006), pp. 365-387.

[16] V. T. Phan, K. Jöchen, A. Melcher, and T. Böhlke. Deep drawing simulations based on microstructural data. In Proceedings in Applied Mathematics and Mechanics, Vol. 10, (2010), pp. 6970.

[17] V. Glavas, T. Böhlke, D. Daniel, and C. Leppin. Texture based finite element simulation of a two-step can forming process. Key Engineering Materials, 504, (2012), pp. 655-660.

[18] T. Maitland and S. Sitzman. Scanning Microscopy for Nanotechnology, Chapter 2: Electron backscatter diffraction (EBSD) technique and materials characterization examples. Springer Science, (2006). pp. 41-75.

[19] F. Bachmann, R. Hielscher, and H. Schaeben. Grain detection from 2d and 3d EBSD dataSpecification of the MTEX algorithm. Ultramicroscopy, 111, (12), (2011), pp. 1720-1733.

[20] R. Hielscher. MTEX quantitative texture analysis software, MTEX version 3.5.0 (2013). http://code.google.com/p/mtex/.

[21] D. Banabic. Sheet metal forming processes: constitutive modelling and numerical simulation. Springer Science \& Business Media, (2010).

[22] S. A. Kim and W. L. Johnson. Elastic constants and internal friction of martensitic steel, ferritic-pearlitic steel, and $\alpha$-iron. Materials Science and Engineering: A, 452, (2007), pp. 633639. 